\title{
Beschäftigung und Mindestlöhne - Neue Ergebnisse der empirischen Mindestlohnforschung
}

Noch vor 15 Jahren gingen die meisten Ökonomen davon aus, dass Mindestlöhne negative Beschäftigungseffekte haben. Die neue empirische Mindestlohnforschung in den USA und in Großbritannien bestätigt diese Annahmen nicht. Angesichts der neuen Fakten hat sich auch das Meinungsbild über Mindestlöhne unter den Ökonomen dieser Länder geändert. In Deutschland allerdings werden diese Ergebnisse systematisch ignoriert und alte Weltbilder zum Mindestlohn durch Modellrechnungen stabilisiert. Es ist an der Zeit, vorliegende internationale Forschungsbefunde sorgfältig zu rezipieren und damit nicht nur den Anschluss an den Stand der Wissenschaft zu halten, sondern auch von ausländischen Erfahrungen zu lernen.

\section{Einleitung}

Der Zusammenhang zwischen Mindestlöhnen und Beschäftigung ist seit Langem Gegenstand heftiger Debatten in der Volkswirtschaftslehre. Der britische Ökonom Manning (2003, S. 338) konstatiert in diesem Zusammenhang, dass viele Ökonomen Schwierigkeiten hätten, auch nur die Möglichkeit in Erwägung zu ziehen, dass Mindestlöhne Beschäftigung nicht zerstören würden - fast so als wolle man gesicherte wissenschaftliche Grundlagen, wie etwa das Gesetz über die Schwerkraft, infrage stellen. Ein Beispiel für ein solches Beharrungsvermögen ist ein gemeinsamer Aufruf der Präsidenten und Direktoren der großen deutschen Wirtschaftsforschungsinstitute vom 12. März 2008 (Blum et al. 2008): „So oder so - der Mindestlohn führt zu erheblichen Beschäftigungsverlusten. Diese Beschäftigungsverluste sind im Westen unseres Landes erheblich. Im Osten werden sie erschütternde Ausmaße annehmen." Auch in anderen entwickelten Ländern teilte lange Zeit die Mehrheit der Ökonominnen und Ökonomen diese Überzeugung. Mittlerweile sind diese Mehrheiten in vielen Ländern durch neue Forschungsergebnisse zu den Beschäftigungswirkungen von Mindestlöhnen brüchig geworden. So forderten im Oktober 2006650 amerikanische Wirtschaftswissenschaftlerinnen und Wissenschaftler, darunter fünf Nobelpreisträger, eine Erhöhung des Mindestlohnes (EPI 2006). Der Meinungsumschwung in den USA und Großbritannien wurde durch neue empirische Untersuchungen, vor allem von Card und Krueger (1995) sowie der britischen Low Pay Commission, angestoßen, die außerdem die lange Jahre festgefahrene Theoriediskussion neu belebten.

Im Folgenden sollen zunächst die theoretischen Argumente für und gegen negative Beschäftigungswirkungen von Mindestlöhnen nachgezeichnet werden (Abschnitt 1). Da sich aus theoretischen Modellen keine eindeutigen Aussagen über die Beschäftigungswirkungen von Mindestlöhnen ableiten lassen, können theoretische Kontroversen nur über empirische Untersuchungen entschieden werden. Daher werden im Anschluss die Ergebnisse wichtiger neuer empirischer Studien zusammengefasst (Abschnitt 2). Der Schwerpunkt wird dabei auf die neue amerikanische Mindestlohnforschung gelegt, vor allem die methodisch innovativen Studien des Institute for Labor and Employment in Berkeley, die bislang noch keinen Eingang in die deutsche Diskussion gefunden haben. ${ }^{1}$ In Abschnitt 3 werden Schlussfolgerungen aus dem neueren Forschungsstand gezogen, die auch für die Mindestlohndebatte in Deutschland zu berücksichtigen sind.

\section{Theorien über den Zusammenhang von Mindestlöhnen und Beschäftigung}

\subsection{DIE NEOKLASSIK}

Die neoklassische Standardargumentation über die Beschäftigungswirkungen von Mindestlöhnen wurde von Stigler (1946) in einem berühmten Aufsatz mit dem Titel „The economics of minimum wage legislation“ entwickelt: In Märkten mit vollkommenem Wettbewerb werden die Beschäftigten nach ihrer Produktivität entlohnt. Bei Einführung eines Mindestlohnes werden die Arbeiter, deren Produktivität unterhalb des Mindestlohnes liegt, entlassen - es sei denn, ihre Produktivität erhöht sich. Dies kann z. B. durch eine höhere Arbeitsintensität oder durch Einführung neuer Technologien erreicht werden. Eine höhere Arbeitsintensität hielt Stigler jedoch bei den gering bezahlten Beschäftigten für unwahrscheinlich, da diese Arbeitskräfte schon durch Armut zu äußerster Anstrengung angetrieben würden und die Arbeitsintensität zur Vermeidung einer Entlassung nochmals um $50 \%$ und mehr - also jenseits des seinerzeit Erwartbaren - gesteigert werden müsse (Stigler 1946, S. 359). Stigler kommt letztlich zu dem Schluss, dass Ökonomen die allgemein geteilte Aussage treffen könnten, dass die Beschäftigungseffekte von Mindestlöhnen negativ seien.

Die wichtigsten Ergebnisse der rund 70 Forschungsberichte zum britischen Mindestlohn wurden an anderer Stelle zusammengefasst (Bosch/ Weinkopf 2006; Bosch/Kalina/Weinkopf 2009).

\footnotetext{
Gerhard Bosch, Prof. Dr., ist geschäftsführender Direktor des Instituts Arbeit und Qualifikation (IAQ), Universität DuisburgEssen. Arbeitsschwerpunkte: Arbeitsmarkt und Beschäftigung, Industrielle Beziehungen Berufliche Bildung. e-mail:gerhard.bosch@uni-due.de
} 
Stigler hat immerhin anerkannt, dass die Beschäftigungseffekte von Mindestlöhnen theoretisch nicht eindeutig determiniert sind, als er auf die Möglichkeit der Steigerung der Produktivität verwies. Gleichwohl schließt er diesen Fall kategorisch aus und kann erst damit eindeutige Aussagen zu den Beschäftigungseffekten ableiten. Stigler hatte den physisch erschöpften Industriearbeiter der 1940er Jahre vor Augen, aus dem keine höhere Leistung herauszuholen sei. Heute aber wissen wir, welche Potenziale der Produktionssteigerung sowohl durch die Reorganisation der Arbeitsabläufe als auch durch die Einführung neuer Technologien seit 1946 realisiert werden konnten. Steigende Löhne waren dabei sicherlich eine Triebkraft der Reorganisation von Unternehmen.

In den 1950er Jahren war die Diskussion über die Wirkung von Mindestlöhnen auf das Beschäftigungsniveau in der Volkswirtschaftslehre noch offen. Preiser (1953) kritisierte etwa die These einer feststehenden Nachfragekurve und einer Entlohnung nach der Grenzproduktivität:

„Die Notwendigkeit einer Ergänzung (der Grenzproduktivitätstheorie - der Autor) ist zunächst offensichtlich, sobald die Frage nach den Möglichkeiten einer dauerhaften Erhöhung der Löhne durch gewerkschaftliche Macht oder staatliches Diktat gestellt wird.... Die Grenzproduktivitätstheorie leugnet diese Möglichkeit; in der Tat wird die relative Seltenheit der Produktivitätsfaktoren dadurch nicht geändert. Eine gewaltsame Erhöhung der Löhne hat daher nur Arbeitslosigkeit zur Folge. Andererseits scheint die Erfahrung zu zeigen, dass solche Lohnerhöhungen möglich sind, ohne dass die Beschäftigung zurückgeht. Gehen sie vielleicht zu Lasten von Monopolrenten, die ohne Gefahr für den Beschäftigungsstand verringert werden könnten? Oder gibt eine solche Lohnerhöhung möglicherweise den Anstoß zu einer gesamtwirtschaftlichen Entwicklung, die alle bisherigen Grundlagen verändert, das Volkseinkommen, den Konsum, die Investitionen, so dass sich nicht nur der Lohn absolut - nominal und real - hebt, sondern auf dem Umweg über verstärkte Investitionen und eine vergrößerte Sachausrüstung der Volkswirtschaft auch die relative Seltenheit des Produktionsfaktors Arbeit und damit seine Quote am Sozialprodukt zunimmt?" (Preiser 1953, S. 279).
In den USA sieht Lester (1964) einen „Bereich der Nichtdeterminiertheit“, in dem man Löhne ändern kann, ohne dass dies nennenswerte Auswirkungen auf die Beschäftigung habe. Der „Schock“ von Lohnerhöhungen könne Unternehmen dazu zwingen, ihre Managementpraktiken und ihre Arbeitsorganisation zu verbessern, wodurch sich die Beschäftigung und der Output erhöhen würden. Lester lenkt den Blick aber auch auf Produktivitätseffekte bei unveränderter Arbeitsorganisation und technischer Ausstattung. Höhere Löhne können die Fluktuation der Beschäftigten verringern, wodurch die Unternehmen nicht nur Rekrutierungs- und Anlernkosten sparen, sondern auch von den Produktivitätssteigerungen durch informelles Lernen profitieren.

In den folgenden Jahrzehnten ist im Mainstream der ökonomischen Theorie der Blick auf diese Handlungsmöglichkeiten und auch auf die von Preiser (1953) angesprochenen makro-ökonomischen Wirkungen zunehmend verloren gegangen. So schreiben Baumol/Blinder (1979) in ihrer Einführung in die Volkswirtschaft: „Die Wirkung von Mindestlöhnen liegt nicht in der Erhöhung des Einkommens der am geringsten qualifizierten Beschäftigten, sondern in der Beschränkung ihrer Beschäftigungsmöglichkeiten." Heilbronner/Thurow (1987) argumentieren: „Mindestlöhne haben zwei Auswirkungen. Sie erhöhen die Verdienste der Beschäftigten, aber sie sind auch die Ursache für den Arbeitsplatzverlust anderer." In der verkürzten Lehrbuchargumentation der Neoklassik wurde die theoretische Offenheit des Ursprungs-Modells von Stigler weitgehend zurückgenommen. Danach werden Beschäftigte in einer Situation vollkommenen Wettbewerbs in Höhe ihrer individuellen Grenzprodukte entlohnt. Reaktionen auf Lohnänderungen wie die Einführung neuer Technologien, eine Verbesserung der Arbeitsorganisation sowie die Weiterbildung und Motivationssteigerung von Beschäftigten - also Maßnahmen, die die Produktivität verbessern können - bleiben in diesem Modell unberücksichtigt. Unternehmer und Beschäftigte werden als reine Anpasser an externe Bedingungen und nicht als aktive und möglicherweise innovative Akteure angesehen. Card/Krue$\operatorname{ger}(1995$, S. 11) merken hierzu ironisch an, dass unter solchen Annahmen die Arbeit der Personalabteilungen sehr einfach sei: Die Personalleiter müssten nur die Markt- löhne beobachten und dementsprechend die Löhne festsetzen.

\subsection{MODIFIKATIONEN DES NEOKLASSISCHEN MODELLS}

In den letzten Jahren konnten in einer Vielzahl von empirischen Untersuchungen keine negativen Auswirkungen von Mindestlöhnen festgestellt werden (vgl. Abschnitt 3). Auf der Suche nach Erklärungen öffnete sich auch die theoretische Debatte wieder. Innerhalb der neoklassischen Theorie wurde auf die Vermachtung von Arbeitsmärkten als mögliche Erklärung hingewiesen. In monopsonistische Arbeitsmarktstrukturen sind die Unternehmen nicht wie im Modell des vollkommenen Wettbewerbs Preisnehmer, sondern verfügen über so viel Marktmacht, dass sie die Löhne unterhalb des Gleichgewichtslohns festsetzen können. Wenn sie ihren Profit maximieren wollen, stellen sie nur solange Arbeitskräfte ein, wie der Grenzerlös die Grenzkosten übersteigt. Unternehmen drosseln auf diese Weise ihre Nachfrage nach Arbeitskräften, um die Löhne niedrig zu halten. Im Ergebnis liegen die gezahlten Löhne unter dem Gleichgewichtslohn und das Beschäftigungsniveau ist auch geringer als bei vollkommenem Wettbewerb. Durch einen extern festgelegten Mindestlohn in Höhe des Gleichgewichtslohnes steigt die Beschäftigung (Card/Krueger 1995, S. 355ff.; OECD 1998, S. 43; Ragacs 2002; Rothschild 1988, S. 40ff.). Die Theorie des Monopsons ist seit Langem bekannt. Sie spielte aber früher keine Rolle, da eine solche Marktmacht eines einzelnen Unternehmens für unwahrscheinlich gehalten wurde. Manning (2003) argumentiert jedoch, dass man sich nicht an dem Präfix "Mono" festhalten sollte. Kein Unternehmen handele völlig isoliert. Man müsse auch an Modelle von Oligopsonen denken, in denen z. B. wenige Unternehmen auf regionalen Arbeitsmärkten die Löhne kontrollieren. Durch die begrenzte Mobilität bestimmter Kategorien von Arbeitskräften steige die regionale Arbeitgebermacht. So könne man zum Beispiel die oft sehr niedrigen Löhne von verheirateten Frauen erklären.

\subsection{KEYNESIANISMUS UND WACHSTUMSTHEORIEN}

In keynesianischen Theorien wird der Doppelcharakter der Löhne berücksichtigt, 
die zugleich sowohl betriebliche Kosten als auch gesamtwirtschaftliche Nachfrage darstellen. Von einer Erhöhung der Reallöhne im unteren Einkommensbereich werden erhebliche Nachfrageeffekte erwartet, da diese Einkommensgruppen eine hohe Konsumneigung aufweisen. Die Arbeitsnachfrage ist weitgehend abhängig von der Güternachfrage. Eine durch die Erhöhung geringer Löhne ausgelöste steigende Güternachfrage erhöht die Nachfrage und somit auch die Beschäftigung (Bartsch 2009, S. 23ff.). ${ }^{2}$ Ausgangspunkt neuerer Wachstumstheorien ist der vielfach nachgewiesene Zusammenhang von wirtschaftlichem Wachstum und Investitionen in Humankapital. Die Kernannahme ist, dass ein Mindestlohn die Anreize für Unternehmen erhöht, in Humankapital zu investieren. In weiteren Mehrgenerationen-Modellen werden zusätzlich die Investitionen von Eltern in die Ausbildung der Kinder thematisiert, die mit höherem Lohn steigen. Cahuc/Michel (1996) zeigen, dass eine Absenkung des Mindestlohnes aus diesem Grund sogar das wirtschaftliche Wachstum verringern kann. Acemoglu/Pischke (1999) machten zudem darauf aufmerksam, dass höhere Löhne den Bestand an Humankapitalinvestitionen und damit das Wachstumspotenzial von Unternehmen erhöhen können, da die Unternehmen die Produktivität der Beschäftigten beispielsweise durch Weiterbildung an das Lohnniveau anpassen.

\subsection{ZWISCHENFAZIT}

Die meisten theoretischen Arbeiten zu Beschäftigungswirkungen von Mindestlöhnen unterstellen ausschließlich Veränderungen der Lohnhöhe und modellieren deren mögliche Auswirkungen auf Beschäftigung unter Abstraktion von Raum, Zeit und institutionellen Rahmenbedingungen. Aus der umfangreichen Literatur zu den Varianten des Kapitalismus (Varieties of Capitalism) (Bosch/Lehndorff/Rubery 2009) wissen wir aber unter anderem, dass die gleiche Intervention in den Arbeitsmarkt in einem Land negative und in einem anderen neutrale oder sogar positive Auswirkungen auf Beschäftigung haben kann - je nachdem, ob durch institutionelle Komplementaritäten mögliche negative Effekte vermieden oder verstärkt werden. Auch Zeit spielt eine große Rolle, da kompensierende und proaktive Maßnahmen, wie die Weiterbildung von Be- schäftigten oder die Reorganisation eines Unternehmens, einen zeitintensiven organisatorischen Vorlauf haben. Man könnte also vermuten, dass ein Mindestlohn in gleicher Höhe völlig unterschiedliche Wirkungen hat, je nachdem, ob er überfallartig eingeführt oder frühzeitig angekündigt wird. Großbritannien hat daher die Erhöhungen des Mindestlohnes immer frühzeitig angekündigt, um den Unternehmen Zeit für Reorganisationen oder Weiterbildung zu geben (Bosch/Weinkopf 2006). Die Wirkungen von Mindestlöhnen werden sich vermutlich auch unterscheiden, je nachdem ob sie auf ein innovationsfreudiges bzw. innovationsfeindliches Umfeld treffen. Deutschland wurde in der oben genannten Literatur zu den Varianten des Kapitalismus als ein Beispiel für ein solches innovationsfreundliches Umfeld hervorgehoben, weil hierzulande durch die Kombination von guter Ausbildung, Kündigungsschutz, Mitbestimmung und langfristiger Orientierung der Unternehmen bei gleichzeitiger Spezialisierung auf Qualitätsprodukte eine hohe Produktivität erzielt werden konnte. In Wirtschaftssystemen mit einem solchen Gleichgewicht hoher Qualifikationen (high skill equilibrium) lassen sich höhere Löhne besser rechtfertigen als in Systemen, die auf niedrigen Qualifikationen (low skill equilibrium) basieren (Finegold/ Soskice 1988).

Die OECD ist von diesen Forschungsergebnissen nicht unbeeindruckt geblieben. Sie verweist daher bei der Bewertung ganz unterschiedlicher Arbeitsmarktinterventionen und -regulierungen in ihrem jährlich erscheinenden Beschäftigungsbericht (Employment Outlook) zunehmend auf die Bedeutung institutioneller Komplementaritäten. Ein bekanntes Beispiel ist die positive Bewertung der großzügigen dänischen Arbeitslosenunterstützung, die trotz einer Lohnersatzrate von $90 \%$ wegen ihrer engen Verknüpfung mit Qualifizierungs- und Aktivierungsstrategien nicht zu der in vielen Lehrbüchern vorausgesagten Erhöhung der Langzeitarbeitslosigkeit führt.

Auf der Basis dieser knappen Erörterung kann man (ähnlich wie OECD 1998) Folgendes festhalten:

(1) Theoretisch lässt sich kein strikter Zusammenhang zwischen Mindestlöhnen und Beschäftigung ableiten. Die Einführung von Mindestlöhnen kann je nach Marktkonstellationen, institutionellem
Umfeld und Reaktionen der Akteure positive oder negative Auswirkungen haben.

(2) Offensichtlich gibt es Spielräume für die Festsetzung von Mindestlöhnen, sodass negative Beschäftigungseffekte erst bei Überschreitung eines bestimmten $\mathrm{Ni}$ veaus erfolgen, während unterhalb dieses Niveaus Mindestlöhne beschäftigungspolitisch neutral sind oder sogar positive Effekte aufweisen.

(3) Die Beschäftigungseffekte hängen von Reaktionen der Akteure ab. In einem hochinnovativen Umfeld, in dem ein Mindestlohn die Unternehmen, die Beschäftigten und auch den Staat zu Innovationen veranlasst, sind die Wirkungen eher positiv als in einer nicht innovativen Umgebung.

(4) Um zeitlichen Spielraum für Verbesserungen der Arbeitsorganisation, Weiterbildung und technologische Innovationen zu schaffen, muss die Einführung oder Erhöhung eines Mindestlohnes frühzeitig angekündigt werden.

(5) Bei einem großen Arbeitskräfteangebot kann ein Mindestlohn zu einer Substitution geringer Qualifizierter durch höher Qualifizierte führen. Dieser Effekt kann jedoch durch gezielte Qualifizierungsanstrengungen für gering Qualifizierte neutralisiert werden.

Offensichtlich ist das „Wie“ bei der Einführung von Mindestlöhnen - also die Höhe und der Einführungsprozess - wichtiger als das „Ob“. Welches Niveau und welche Implementationsbedingungen notwendig sind, um negative Beschäftigungseffekte $\mathrm{zu}$ vermeiden, lässt sich nur empirisch feststellen.

\section{Ergebnisse der neueren Mindestlohnforschung}

\subsection{MODELLRECHNUNGEN}

Zunächst einmal ist es notwendig, genauer zu bestimmen, was unter empirischen Untersuchungen zu den Beschäftigungseffek-

2 Diese Diskussionslinie wird detaillierter im Beitrag von Daniel Detzer in diesem Heft verfolgt. 
ten von Mindestlöhnen zu verstehen ist. So zählen beispielsweise Bachmann et al. (2008) auch Simulationsrechnungen zu empirischen Untersuchungen. Alle in die Zukunft gerichteten Schätzungen und Prognosen, wie methodisch anspruchsvoll sie auch sein mögen, bleiben jedoch Modellrechnungen und sind nicht der harte empirische Test unterschiedlicher aus der Theorie abgeleiteter Hypothesen. Vor allem aber können die Ergebnisse solcher Schätzungen immer von ihren Autorinnen und Autoren durch die Auswahl ihrer Annahmen gestaltet werden.

Ragnitz/Thum (2007) legen ihrer Berechnung eine negative Lohnelastizität von minus 0,75 zugrunde. Dies bedeutet, dass eine Erhöhung des Lohnes um 1 \% zu einer Verringerung der Beschäftigung von $0,75 \%$ führt. Unter dieser Annahme führt ein Mindestlohn in Höhe von 7,50 € zu einem Verlust von 1,1 Mio. Beschäftigungsverhältnissen. Bachmann et al. (2008) kommen in ihren Modellrechnungen sogar zu einem Verlust von 1,22 Mio. Arbeitsplätzen bei Einführung eines Mindestlohnes von $7,50 €$. Zwei Studien des Deutschen Instituts für Wirtschaftsforschung (DIW) rechnen mit negativen Beschäftigungseffekten von 141.000 bzw. 291.000 Personen (Müller 2009; Müller/Steiner 2008). Überraschend ist die hohe Streubreite der Berechnungen. Der höchste Wert liegt fast neunmal höher als der niedrigste. Hüther (2008) vom Institut der deutschen Wirtschaft (IW) sieht ohne tiefere Begründung 4 Mio. Arbeitsplätze gefährdet. Wenn man diesen Wert mit einbezieht, steigt die Spannbreite auf eine Relation von eins zu achtundzwanzig.

Die Modelle von Ragnitz/Thum (2007) sowie von Bachmann et al. (2008) beinhalten nicht nur hohe negative Lohnelastizitäten, wie sie in den empirischen Untersuchungen zu Mindestlöhnen selbst für gering Qualifizierte oder Teenager nicht gefunden wurden. Hier liegen die Werte meistens bei 0,2 (Neumark/Wascher 2007a). Vielmehr werden auch Nachfragewirkungen erhöhter Mindestlöhne ausgeblendet. Unter Berücksichtigung der Nachfragewirkungen sowie realistischer Lohnelastizitäten kommt Bartsch (2009) sogar zu positiven langfristigen Beschäftigungswirkungen von rund 100.000 Personen. Diese Berechnungen sind deutlich realitätsnäher. Ein „Beweis“ für positive Effekte sind sie jedoch auch nicht. Einen solchen Beweis kann letztlich nur echte Empirie liefern.
Dazu schrieben Burkhauser/Finegan (1993, S. 123), dass 40 Jahre empirische Forschung keinen Zweifel daran lassen, dass Mindestlöhne die Beschäftigung reduzieren. Diese Auffassung ist zwar auch heute noch weit verbreitet, kann aber durch eine Vielzahl neuerer empirischer Studien als widerlegt gelten.

\subsection{EMPIRISCHE FORSCHUNGS- BEFUNDE}

\section{USA UND FRANKREICH}

Card und Krueger (1994, 1995) haben mit ihren Studien den Grundstein für eine differenzierte Sicht gelegt. Diese beiden Autoren untersuchten die Entwicklung der Beschäftigung in der Fast-Food-Branche in den benachbarten US-Bundesstaaten New Jersey und Pennsylvania nach der deutlichen Erhöhung des Mindestlohnes in New Jersey. ${ }^{3}$ Fast Food-Restaurants gelten als der Prototyp von lohnintensiven Branchen mit hohem Preiswettbewerb und leicht austauschbaren Arbeitskräften, die sehr elastisch auf Lohnerhöhungen reagieren. In diese „quasi-experimentelle“ Untersuchung wurden 331 Fastfood-Restaurants in New Jersey und 79 in Pennsylvania einbezogen. Der Vergleich zeigte, dass die Beschäftigung in New Jersey trotz der Erhöhung des Mindestlohnes nicht zurückging, sondern sogar etwas stärker anstieg als in Pennsylvania.

Nun kann man mit Recht fragen, ob die gleichen Wirkungen der Anhebung eines relativ niedrigen Mindestlohnes in einem US-amerikanischen Staat auch in US-Staaten mit höheren Mindestlöhnen zu beobachten sind. Card/Krueger (1995) haben dies nicht nur in mehreren eigenen Untersuchungen ${ }^{4}$ bestätigen können, sondern auch eine neue Welle „quasiexperimenteller" Forschungen in den USA, vor allem im Institute for Labour and Employment in Berkeley, angeregt. Mit "quasi-experimentell“ ist der Vergleich der Auswirkungen von Mindestlöhnen auf die Beschäftigung in vergleichbaren realen Fällen mit unterschiedlichen Mindestlöhnen gemeint. Wie wir sehen werden, liegt das größte Problem dieser Vorgehensweise darin, die Vergleichbarkeit dieser realen Fälle sicherzustellen.

Dube et al. (2008) vergleichen Beschäftigungs- und Lohndaten in 318 aneinandergrenzenden Paaren von Counties, die jeweils zu einem anderen Bundesstaat gehören. Sie nehmen an, dass diese Counties wegen ihrer ähnlichen Wirtschaftstruktur eher miteinander vergleichbar sind als die größeren Bundesstaaten mit ihren sehr heterogenen Wirtschaftstrukturen und ökonomischen Entwicklungen. Sie unterscheiden weiter zwischen Paaren, in denen nur der nationale Mindestlohn galt, es also keine Lohnunterschiede gab, und zwischen Vergleichspaaren mit unterschiedlichen Mindestlöhnen. ${ }^{5}$ Die Unterschiede im Mindestlohn zwischen diesen Vergleichspaaren lagen zwischen 1990 und 2006 zwischen $7 \%$ und $20 \%$, wobei die Unterschiede in den letzten Jahren gewachsen sind. Die anderen 1.380 Counties, die nicht zu den Vergleichsgruppen gehörten, wurden als Kontrollgruppe beibehalten. Die Studie zeigt, dass höhere Mindestlöhne in einem Vergleichspaar die tatsächlichen Verdienste der Beschäftigten in der Gastronomie, aber auch in anderen typischen Niedriglohnbranchen, deutlich erhöhen, ohne dass es zu negativen Beschäftigungseffekten kommt.

Dieses Ergebnis steht im Gegensatz zu früheren Arbeiten von Neumark/Wascher (1992, 2007b), die als Vergleichspaare Bundesstaaten auswählten. Dube et al. (2008) sehen in den großräumigen Vergleichen von Neumark/Wascher die Gefahr einer Verzerrung durch „nicht beobachtete Heterogenität“. Damit ist gemeint, dass bei nationalen Vergleichen der Beschäftigungsentwicklung in einzelnen Staaten ganz andere Faktoren die Beschäftigungsentwicklung bestimmen als die Mindestlöhne. So kann die Beschäftigungsentwicklung im Süden der USA durch die Entwicklung des Tourismus positiv und im sogenannten „rust-belt“ durch die Krise des verarbeitenden Gewerbes negativ beeinflusst werden, ohne dass es etwas mit der Höhe der Mindestlöhne zu tun hat.

31992 erhöhte New Jersey den Mindestlohn um fast $20 \%$ auf 5,05 \$, während im benachbarten Pennsylvania der Mindestlohn bei 4,25 \$ konstant blieb.

4 Sie untersuchten etwa die Auswirkungen der Erhöhung des nationalen Mindestlohnes auf die Beschäftigung in Bundesstaaten mit niedrigen und solchen mit höheren Löhnen. In Bundesstaaten mit niedrigen Löhnen war der relative Lohnanstieg größer als in den Hochlohnstaaten. Tatsächlich sind die Löhne im unteren Bereich gestiegen, ohne dass dies jedoch die Beschäftigung verringerte.

5 Die amerikanischen Bundesstaaten können für ihren Staat den nationalen Mindestlohn über das nationale Mindestniveau anheben. Auch Städte und Gemeinden haben diese Möglichkeit. 
Bei benachbarten Vergleichspaaren von Counties aus unterschiedlichen Staaten, also kleinen räumlichen Einheiten, sind diese Unterschiede erheblich verringert. Wenn Dube et al. (2008) ihre Kontrollpaare aufgeben und zu den groben Vergleichen von Neumark/Wascher zurückkehren, kommen sie hingegen zu negativen Beschäftigungswirkungen. Dies zeigt, wie anfällig die empirische Forschung zu Mindestlöhnen für unterschiedliche methodische Spezifikationen ist.

Bislang haben wir nur über Untersuchungen zu den einzelstaatlichen Mindestlöhnen in den USA berichtet, die im Vergleich zu den westeuropäischen Mindestlöhnen auf einem relativ niedrigen Niveau liegen. Viele amerikanische Städte haben aber inzwischen deutlich höhere Mindestlöhne für ihren Geltungsbereich festgelegt. ${ }^{6}$ Diese sogenannten „living wages“, die z.T. nur für öffentliche Aufträge gelten, orientieren sich an den Lebenshaltungskosten, die in den Großstädten deutlich über dem Durchschnittsniveau liegen (Reich 2003, S. 205). So wurde der Mindestlohn in San Francisco 2004 auf 8,50\$, 2007 auf 9,14 \$ und 2009 auf 9,79\$ angehoben. Auftragnehmer der Stadt müssen 2009 sogar einen Mindestlohn von 11,54 \$ zahlen.

Dube et al. (2007) untersuchten die Wirkungen der Anhebung der „living wages" in den Jahren 2004 und 2007. Sie bildeten wieder Vergleichspaare in San Francisco und Umgebung, analysierten aber diesmal nicht nur die Wirkungen auf die Beschäftigung im Gastronomiebereich, sondern auch auf Arbeitszeit, Krankenversicherung, Preise, Betriebszugehörigkeit und Trinkgelder. Zudem wurde die Beschäftigung in den Touristenvierteln in San Francisco mit der Entwicklung in anderen Stadteilen verglichen, um eventuelle Sondereffekte aufzuspüren. Die „living wages" erhöhten die Löhne im unteren Bereich deutlich, selbst in den chinesischen Restaurants, die als kritischer Fall galten. Der Anteil der Beschäftigten, die weniger als 8,50 \$ erhielten, verminderte sich von $50 \%$ auf $5 \%$. Es wurden weder höhere Raten von Betriebsschließungen noch Beschäftigungsabbau festgestellt. Vor allem in den Fastfood-Restaurants erhöhten sich der Anteil der Vollzeitbeschäftigten und die Betriebszugehörigkeit. Die Preise wurden nur leicht angehoben. Die Qualität der Beschäftigung glich sich der in Bedienungsrestaurants an. Es gab keine Anzeichen, dass zum Ausgleich der Lohnerhöhungen die Zahlungen für Krankenversicherung verringert wurden.

Die Hinweise, dass eine Anhebung der unteren Löhne die Geschäftsmodelle von Unternehmen nachhaltig verändern kann, sind noch klarer in der Flughafenstudie von Reich et al. (2003) zu erkennen. Nach der Deregulierung der Flugindustrie 1978 hatten alle Fluggesellschaften viele Tätigkeiten zur Verringerung der Löhne ausgelagert, sodass ein großer Niedriglohnsektor entstanden ist. Etwa ein Drittel der 30.000 Beschäftigten in 140 Unternehmen verdiente weniger als $10 \$$ pro Stunde. Die Flughafenkommission in San Francisco beschloss 2001 ein Qualitätsprogramm, das einen Mindestlohn von 10 \$ ohne Sozialleistungen und 11,25 \$ mit Sozialleistungen und zudem ein Anrecht auf 40 Stunden Qualifizierung vorsah. 9.700 Beschäftigte erhielten eine Lohnerhöhung, darunter 5.400 , die zuvor weniger als 10 \$ pro Stunde bekommen hatten. Die Einstiegslöhne für einfache Tätigkeiten wurden um $33 \%$ angehoben. Alle Beschäftigten sind nun krankenversichert. Die Löhne von weiteren 2.550 Beschäftigten, die nicht direkt unter das Programm fielen, aber am Flughafen tätig waren, wurden ebenfalls erhöht. Die direkten Kosten des Programms lagen bei 42,7 Mio. \$ pro Jahr. Diese Kosten wurden teilweise wieder direkt eingespielt durch die Verringerung der Fluktuation. Diese fiel um $30 \%$ in allen Unternehmen und um $60 \%$ in den Unternehmen, die die Löhne um mehr als $10 \%$ erhöhten. Der größte Rückgang der Fluktuation von 94,7 \% im April 2000 auf 18,7 \% im September 2001 war beim Personal der Sicherheitskontrollen zu verzeichnen. Allein hierdurch wurden 6,6 Mio. \$ pro Jahr eingespart. Die Unternehmen berichten weiterhin, dass die Qualität der Arbeit gestiegen sei und sich Fehlzeiten sowie Beschwerdeeingaben (grievances) der Beschäftigten und Disziplinarmaßnahmen gegenüber Beschäftigten verringert hätten.

In einer Übersicht über die Mindestlohnforschung wies die OECD (1998, S. 47f.) auf - allerdings geringe - Beschäftigungsrisiken für Jugendliche hin. So sei z.B. in Frankreich die Beschäftigungsquote von Teenagern zwischen 1975 und 1996 um 18,5 \% gesunken. Davon waren aber nur 0,3 \% auf die Erhöhung des Mindestlohnes, der Rest auf andere Faktoren wie den Ausbau des Bildungssystems zurückzuführen. Untersuchungen zu den
Wirkungen des Mindestlohnes in Frankreich auf die Beschäftigung Jugendlicher kommen zu unterschiedlichen Ergebnissen, die von leicht positiven bis zu leicht negativen Beschäftigungseffekten reichen (Sturn 2008). Auch in den USA zeigte eine Reihe von Untersuchungen negative Beschäftigungseffekte von Mindestlöhnen für Teenager. Offensichtlich hängen die Ergebnisse in beiden Ländern sehr stark von den methodischen Spezifikationen ab.

Allegretto et al. (2008) gingen in der Kontrolle der unbeobachteten räumlichen Heterogenität noch einen Schritt über Dube et al. (2008) hinaus. Sie verglichen die Beschäftigungsentwicklung von Teenagern in Vergleichspaaren von Counties aus unterschiedlichen Staaten, die vom Bureau of Labor Statistics als Pendlerregionen identifiziert wurden. Der Vergleich beschränkt sich somit auf Regionen, die durch Pendlerströme ökonomisch unmittelbar miteinander verbunden sind. Die Autoren zeigen, dass höhere Mindestlöhne die Beschäftigung von Teenagern nicht negativ beeinflussen. Sie finden sogar positive Effekte, die allerdings statistisch nicht signifikant sind. Sobald sie diesen detaillierten Vergleich aufgeben und Counties in den USA mit unterschiedlichen Mindestlöhnen miteinander vergleichen, werden die traditionellen negativen Effekten reproduziert. Selbst diese liegen jedoch mit rund 0,15 noch weit unter den negativen Lohnelastizitäten, mit denen Bachmann et al. (2008) und Ragnitz/Thum (2007) rechnen.

\section{DEUTSCHLAND}

Die bislang einzige empirische Studie zu Mindestlöhnen in Deutschland wurde von König/Möller (2007) vorgelegt. Sie untersuchen den Einfluss des Mindestlohnes in der Bauwirtschaft nach dem Arbeitnehmer-Entsendegesetz. In ihrem „quasiexperimentellen“ Ansatz vergleichen sie die Beschäftigungsentwicklung von Arbeitnehmern mit Mindestlohn und einer Vergleichsgruppe mit einem leicht höheren Verdienst. Sie kommen zu leicht positiven (allerdings nicht oder nur schwach signifikanten) Effekten für Westdeutschland und zu negativen Effekten in Ostdeutschland. Das Studiendesign ist überzeugend. Das eigentliche Problem der Studie liegt in der Datenlage. Ein Teil der Beschäftigten, die

62003 waren es fast 100 US-amerikanische Städte und Gemeinden (Reich 2003, S. 199). 
Abb. 1: Beschäftigungsquoten insgesamt und mit einer Qualifikation unterhalb Sekundarstufe II - in \% -

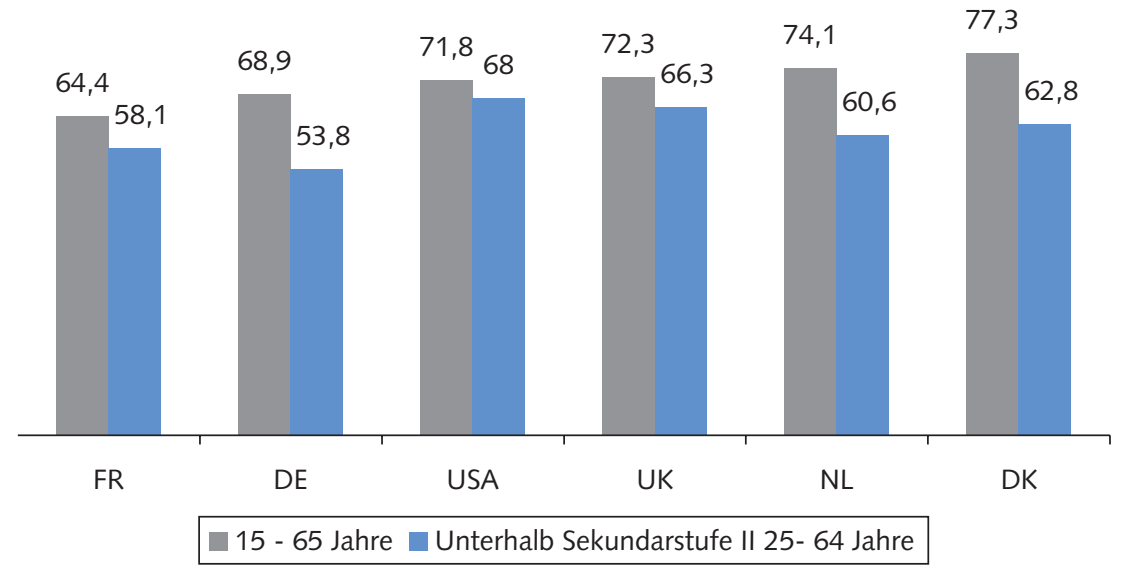

Quelle: OECD 2008.

WSI MITTELLUNGEN

den Mindestlohn erhalten, sind ausländische Werkvertragsnehmer, die in den deutschen Lohn- und Gehaltsstatistiken nicht auftauchen. Verlagerungen zwischen heimischen Arbeitskräften und Werkvertragsnehmern können damit nicht erfasst werden. Zudem ist nicht klar, ob der Mindestlohn für Werkvertragsnehmer wirklich gezahlt wird. Die Verstöße in diesem „grauen Markt" sind beachtlich (Bosch/ Worthmann 2006). Umso überraschender sind die positiven Effekte in Westdeutschland und die nur leicht negativen Effekte in Ostdeutschland mit seiner insgesamt prekären Beschäftigungssituation zum Untersuchungszeitpunkt.

\section{LÄNDERVERGLEICHE}

Die institutionellen Komplementaritäten, die negative Wirkungen von Mindestlöhnen kompensieren können, stehen im Vordergrund eines Sechs-Ländervergleichs (Dänemark, Deutschland, Frankreich, Großbritannien, Niederlande und die USA) (Bosch 2009; Gautié/Schmitt 2010). Von diesen sechs Ländern hat Dänemark mit einem tariflich vereinbarten Mindestlohn von $13,80 €$ (12€ plus $1,80 €$ Urlaubsgeld) die weitaus höchste Mindestlohnschwelle und gemeinsam mit Frankreich den niedrigsten Anteil an gering Bezahlten. Der Mindestlohn in Dänemark wird zudem noch durch eine Lohnersatzrate von $90 \%$ bei Arbeitslosigkeit stabilisiert. Nach den neoklassischen Arbeitsmarktmodellen müsste dies zu hoher Arbeitslosigkeit vor allem bei gering Qualifizierten führen. Dänemark hat jedoch trotz seines hohen Mindestlohnes nicht nur die höchste Beschäftigungsquote aller Vergleichsländer, sondern auch die zweithöchste Beschäftigungsquote der gering Qualifizierten im Ländervergleich (Abbildung 1).

Aufgrund des guten Bildungssystems ist zudem die Gruppe der gering Qualifizierten kleiner als in den anderen Ländern. Eine Ursache für die hohe Beschäftigungsquote der gering Qualifizierten in Dänemark sind höhere Investitionen in die Weiterbildung (Tabelle 1). Auch Frankreich gelingt es, mit Hilfe seines nationalen

\section{Tabelle 1: Anzahl der zu erwartenden Weiterbildungsstunden für 25- bis 65-Jährige in nicht formaler Weiterbildung nach Bildungsniveau 2003 (pro Kopf im Lebensverlauf)}

\begin{tabular}{lccc}
\hline Land & Unterhalb Sekundarstufe II & Sekundarstufe II & Tertiär \\
\hline Dänemark & 719 & 836 & 1230 \\
Deutschland & 130 & 390 & 650 \\
Frankreich & 450 & 692 & 1061 \\
Niederlande & 216 & 308 & 322 \\
Großbritannien & 103 & 297 & 480 \\
USA & - & 374 & 746 \\
\hline \multirow{2}{*}{ Quelle: OECD 2008a, Table C5.1a. } & & WS MITTEILUNGEN
\end{tabular}

Weiterbildungsfonds höhere Investitionen in die betriebliche Weiterbildung gering Qualifizierter anzuregen als die anderen Länder mit höheren Niedriglohnanteilen. Die potenziell negativen Beschäftigungseffekte höherer Mindestlöhne können somit durch die positiven Wirkungen von Ausund Weiterbildung kompensiert werden.

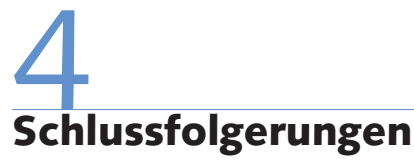

Die neuere empirische Mindestlohnforschung zeigt, dass nicht nur sehr niedrige Mindestlöhne, wie die Mindestlöhne in vielen US-Staaten, beschäftigungsneutral sind, sondern auch die Mindestlöhne in westeuropäischen Staaten, die von mehr als $8 €$ bis $13,80 €$ (Dänemark) reichen, oder die „living wages“ in US-amerikanischen Städten, die in Kaufkraft gemessen bei $10 €$ und mehr liegen. Es ist somit nicht mehr möglich, die Forschungsergebnisse wegen der geringen Höhe der untersuchten Mindestlöhne als für Deutschland irrelevant zu qualifizieren.

Mindestlöhne können die Geschäftsmodelle von Unternehmen nachhaltig beeinflussen. Sie können dazu beitragen, die im Niedriglohnbereich oft sehr hohe Fluktuation so weit zu verringern, dass sich für Unternehmen Investitionen in Weiterbildung und auch eine Erweiterung der Arbeitsinhalte wieder lohnen. Gleichzeitig verringern sich die Aufwendungen für die Kontrolle der Beschäftigten, die besser motiviert sind und eigenständiger arbeiten. Es gibt starke Hinweise auf monopsonistische Arbeitsmarkstrukturen in Niedriglohnbereichen. Durch Mindestlöhne können Extraprofite abgebaut und marktgerechte Löhne gesichert werden.

Länder mit einem institutionellen Umfeld, das Weiterbildung, Modernisierung der Arbeitsorganisation und Innovation unterstützt, können sich höhere Mindestlöhne eher „leisten“ als Länder ohne solche positiven Rückkoppelungen. Das in Deutschland häufig verwendete Argument, die britischen und US-amerikanischen Forschungsergebnisse kämen vor allem wegen des niedrigen Regulierungsniveaus der dortigen Arbeitsmärkte nicht zu negativen Beschäftigungseffekten, konnte nicht bestätigt werden. Es spricht hingegen vieles dafür, dass Mindestlöhne in deregulierten Arbeitsmärkten wegen unzureichender in- 
stitutioneller Unterstützung etwa von Weiterbildung niedriger als in Arbeitsmärkten mit pro-aktiven Regulierungen sind.

Die größte Schwäche der neoklassischen Marktmodelle sind die statischen Annahmen über die Produktivität der Beschäftigten und die Innovationsfähigkeit der Unternehmen. Die Annahme, dass Unternehmen reine Preisanpasser seien und die Produktivität von Beschäftigten ein unverrückbares individuelles Merkmal sei, entspricht nicht der Realität dynami- scher Märkte und innovativer Unternehmer. Zudem bleibt unberücksichtigt, dass in den vernetzten modernen Unternehmen das Produktionsergebnis weitgehend ein Produkt gemeinsamer Arbeit ist. Der in der Volkswirtschaftstheorie unterstellte Fall abgrenzbarer Einzelproduktivitäten ist eher die Ausnahme.

Aus der internationalen Forschung kann man folgende Schlussfolgerungen für Deutschland ziehen: Da Unternehmer nicht reine Marktanpasser sind, müssen erstens Mindestlöhne frühzeitig angekündigt werden, damit Unternehmen Zeit für die eventuell notwendige Reorganisation haben. Zweitens sollte die Einführung von Mindestlöhnen durch eine aktive Qualifizierungs- und Innovationspolitik begleitet werden, um die Produktivität der Beschäftigten anzuheben. Drittens sollte man bei der Festsetzung von Mindestlöhnen vorsichtig einsteigen und dann die Mindestlöhne schrittweise auf das westeuropäische Niveau erhöhen.

\section{LITERATUR}

Acemoglu, D./Pischke, J.-S. (1999): Beyond Becker. Training in Imperfect Labour Markets, in: Economic Journal 109, S. 112-142

Allegretto, S./Dube, A./Reich, M. (2008): Do Minimum Wages Really Reduce Teen Employment? Accounting for Heterogeneity and Selectivity in State Panel Data Center on Wage and Employment Dynamics, Research Paper

Bachmann, R./Bauer, T. K./Kluve, J. (2008): Mindestlöhne in Deutschland. Beschäftigungswirkungen und fiskalische Effekte, RWI-Materialien 43, Essen

Bartsch, K. (2009): Was bringt ein gesetzlicher Mindestlohn für Deutschland? Eine aktualisierte und erweiterte Simulationsstudie zu den gesamtwirtschaftlichen Effekten der Einführung eines allgemeinverbindlichen gesetzlichen Mindestlohnes auf der Basis der Konzeption der Vereinigten Dienstleistungsgewerkschaft ver.di und der Gewerkschaft Nahrung-Genuss-Gaststätten (NGG), Neuendorf

Baumol, W. J./Blinder, A. S. (1979): Economics: Principles and Policy, New York et al.

Blum, U./Hüther, M./Schmidt, C. M./Sinn, H.-W./Snower, D. J./ Straubhaar, T./Zimmermann, K. F. (2008): Gemeinsamer Aufruf der Präsidenten und Direktoren der Wirtschaftsforschungsinstitute vom 12. März 2008, in: ifo Schnelldienst 6 "Mindestlohn: Für und Wider", S. 3-4

Bosch, G. (2009): Low wage work in five European countries and the US, in: International Labour Review 4, S. 337-356

Bosch, G./Weinkopf, C. (2006): Mindestlöhne in Großbritannien: ein geglücktes Realexperiment, in: WSI-Mitteilungen 3, S. 125-130 Bosch, G./Kalina, T./Weinkopf, C. (2009): Mindestlöhne - Hat Deutschland aus den britischen Erfahrungen gelernt?, in: Urbach, K./ Fahrmeir, A. (Hrsg.): Industrieentwicklung: Ein deutsch-britischer Dialog - The Promotion of Industry: An Anglo-German Dialogue, PrinzAlbert-Studien 27, München, S. 115-136

Bosch, G./Lehndorff, S./Rubery, J. (Hrsg.) (2009): European Employment Models in Flux: A Comparison of Institutional Change in Nine European Countries, Basingstoke

Bosch, G./Worthmann, G. (2006): Arbeitsmobilität in der EU - bisherige Erfahrungen und künftige Herausforderungen, in: Arbeit 15, S. 292-297

Burkhauser, R. V./Finegan, T. A. (1993): The economics of minimum wage legislation revisited, in: The Cato Journal 1, S. 123-129
Cahuc, P./Michel, P. (1996): Minimum wage unemployment and growth, in: European Economic Review 7, S. 1463-1482

Card, D./Krueger, A. B. (1994): Minimum Wages and Employment: A Case Study of the Fast-Food Industry in New Jersey and Pennsylvania, in: The American Economic Review 4, S. 772-793

Card, D./Krueger, A. B. (1995): Myth and Measurement: The New Economics of the Minimum Wage, Princeton

Card, D./Krueger, A. B. (2000): Minimum Wages and Employment: A Case Study of the Fast-Food Industry in New Jersey and Pennsylvania: Reply, in: The American Economic Review 5, S. 1397-1420

Draca, M./Machin, S./van Reenen, J. (2006): Minimum wages and Profitability, IZA Discussion Paper 1913, Bonn

Dube, A./Lester, T. W./Reich, M. (2008): Minimum Wage Effects Across State Borders: Estimates Using Contiguous Counties, Institute for Labor and Employment Working Paper Series, University of California, Berkeley

Dube, A./Naidu, S./Reich, M. (2007): The Economic Effects of a Citywide Minimum Wage, in: Industrial \& Labor Relations Review 4, S. $522-543$

Economic Policy Institute (EPI) (2006): EPI on the minimum wage. EPI-news 27, Washington (including: Hundreds of Economists Say: Raise the Minimum Wage)

Finegold D./Soskice D. (1988): The Failure of Training in Britain: Analysis and Prescription, in: Oxford Review of Economic Policy 4, S. 21-52

Gautié, J./Schmitt, J.(2010) (Hrsg.): Low-wage work in the wealthy world, New York

Heilbronner, R. L./Thurow, L. C. (1987): Economics explained, New York

Hüther, M. (2008): Mindestlohn-Pläne gefährden vier Millionen Jobs, http://www.rp-online.de/public/article/politik/deutschland/520546/ Mindestlohn-Plaene-gefaehrden-vier-Millionen-Jobs.html

König, M./Möller J. (2007): Mindestlohneffekte des Entsendegesetzes? Eine Mikrodatenanalyse für die deutsche Bauwirtschaft, in: IAB Discussion Paper 790, Nürnberg

Lester, R. A. (1964): The Economics of Labor, New York et al. Low Pay Commission (1998): The National Minimum Wage. First Report of the Low Pay Commission, The Stationary Office, London, http://www.dti.gov.uk/er/lowpay/ 
Machin, S./Manning, A./Rahman, L. (2003): Where the Minimum Wage Bites Hard: the Introduction of the UK National Minimum Wage to a Low Wage Sector, in: Journal of the European Economic Association 1, S. 154-180

Manning, A. (2003): Monopsony in Motion: Imperfect Competition in Labor Markets, Princeton

Metcalf, D. (2007): Why has the British National Minimum Wage had Little or No Impact on Employment?, CEPR Discussion Paper 781 Müller, K.-U. (2009): Wie groß sind die Beschäftigungsverluste eines allgemeinen Mindestlohnes?, in: DIW-Wochenbericht 26, S. 430-433 Müller, K.-U./Steiner, V. (2008): Mindestlöhne kosten Arbeitsplätze: Jobverluste vor allem bei Geringverdienern, in: DIW-Wochenbericht 30, S. $418-423$

Neumark, D./Wascher, W. (1992): Employment Effects of Minimum and Subminimum Wages: Panel Data on State Minimum Wage Laws, in: Industrial and Labor Relations Review 1, S. 55-81

Neumark, D./Wascher, W. (1995): The Effect of New Jersey's Minimum Wage increase on Fast-Food Employment: A Re-Evaluation using Payroll Records, in: National Bureau of Economic Research, Working Paper Series 5224

Neumark, D./Wascher, W. (2000): Minimum Wages and Employment: A Case Study of the Fast-Food Industry in New Jersey and Pennsylvania: Comment, in: American Economic Review 5, S. 1362-1396 Neumark, D./Wascher, W. (2007a): Minimum Wages and Employment IZA DP 2570, Bonn

Neumark, D./Wascher, W. (2007b): Minimum Wages, the Earned Income Tax Credits and Employment: Evidence from Post-Welfare Reform Era, NBER Working Paper 12915, Cambridge, Mass.
OECD (1998): Making most of the Minimum: Statutory Minimum Wages, Employment and Poverty, in: Employment Outlook, Paris, S. $31-79$

OECD (2008a): Education at a Glance, Paris

OECD (2008b): Employment Outlook, Paris

Preiser, E. (1953): Erkenntniswert und Grenzen der Grenzproduktivitätstheorie, in: Schweizerische Zeitschrift für Volkswirtschaft und Statistik 89, S. 25-45

Ragacs, C. (2002): Warum Mindestlöhne die Beschäftigung nicht reduzieren müssen. Neoklassische Ansätze im Überblick, in: Wirtschaft und Gesellschaft 1, S. 59-84

Ragacs, C. (2003): Mindestlöhne und Beschäftigung: Die empirische Evidenz. Ein Literaturüberblick, in: Wirtschaft und Gesellschaft 2, S. 215-246 Ragnitz, J./Thum, M. (2007): Zur Einführung von Mindestlöhnen: Empirische Relevanz des Niedriglohnsektors, in: Ifo Dresden berichtet 3, S. 36-39

Reich, M. (2003): Living wage ordinances in California, in: The State of California Labor, S. 199-226

Reich, M./Hall, P./Jacobs, K. (2003): Living Wages and Economic Performance: the San Francisco Airport Model, Institute of Industrial Relations, University of California, Berkeley

Rothschild, K. (1988): Theorien der Arbeitslosigkeit, München/Wien Stigler, G. J. (1946): The Economics of Minimum Wage Legislation, in: American Economic Review 3, S. 358-365

Sturn, S. (2008): Beschäftigungseffekte des französischen Mindestlohns, in: Horn, G./Joebges, H./Logeay, C./Sturn, S. (2008): Frankreich: Ein Vorbild für Deutschland? Ein Vergleich wirtschaftspolitischer Strategien mit und ohne Mindestlohn, IMK Report 31, S. 22-25 